\title{
THE DUAL BURDEN OF TUBERCULOSIS AND DIABETES IN HOSPITALIZED SPANISH PATIENTS
}

\author{
A. Durán', A. Ortolá ${ }^{1}$, I. Crespo', B. Calvo², M. Méndez², J. Marco². \\ 1 Endocrinology and Nutrition Department, Hospital Clínico San Carlos, Madrid, Spain. \\ 2 Internal Medicine Department, Hospital Clínico San Carlos, Madrid, Spain.
}

\section{Introduction}

The dual burden of Tuberculosis (TBC) and Diabetes Mellitus (DM) has attracted much attention in the past decade.

Patients with Type 2 Diabetes have a higher risk of developing pulmonary tuberculosis. Moreover, DM comorbidity in pulmonary TB is associated with poor treatment outcomes. Patients admitted to Internal Medicine Departments are an ideal setting to analyze the prognostic roll of DM on TBC clinical course.

\section{Material and methods}

The Minimum Basic Data Set (MBDS) from all discharges of Internal Medicine patients from public hospitals in Spain between 2005 and 2011 was analyzed. All patients with a TBC diagnosis were selected (codes CIE 9-MC), comparing those with and without DM. The adjusted Charlson index was used as a measure of patient comorbidity.

Univariate and bivariate statistical analysis was performed where a $p<0.005$ was considered significant.

\section{Results}

A total of 25,367 patients with TBC were identified; $9.2 \%$ of them had also a diagnosis of DM2 (Figure 1).

Patients with DM 2 had a higher mortality than non-DM patients $(7.7 \%$ vs $4.8 \%$; p<0.01) (Figure 2).

Hypoglycaemia was registered in 150 cases, also associated with higher mortality.

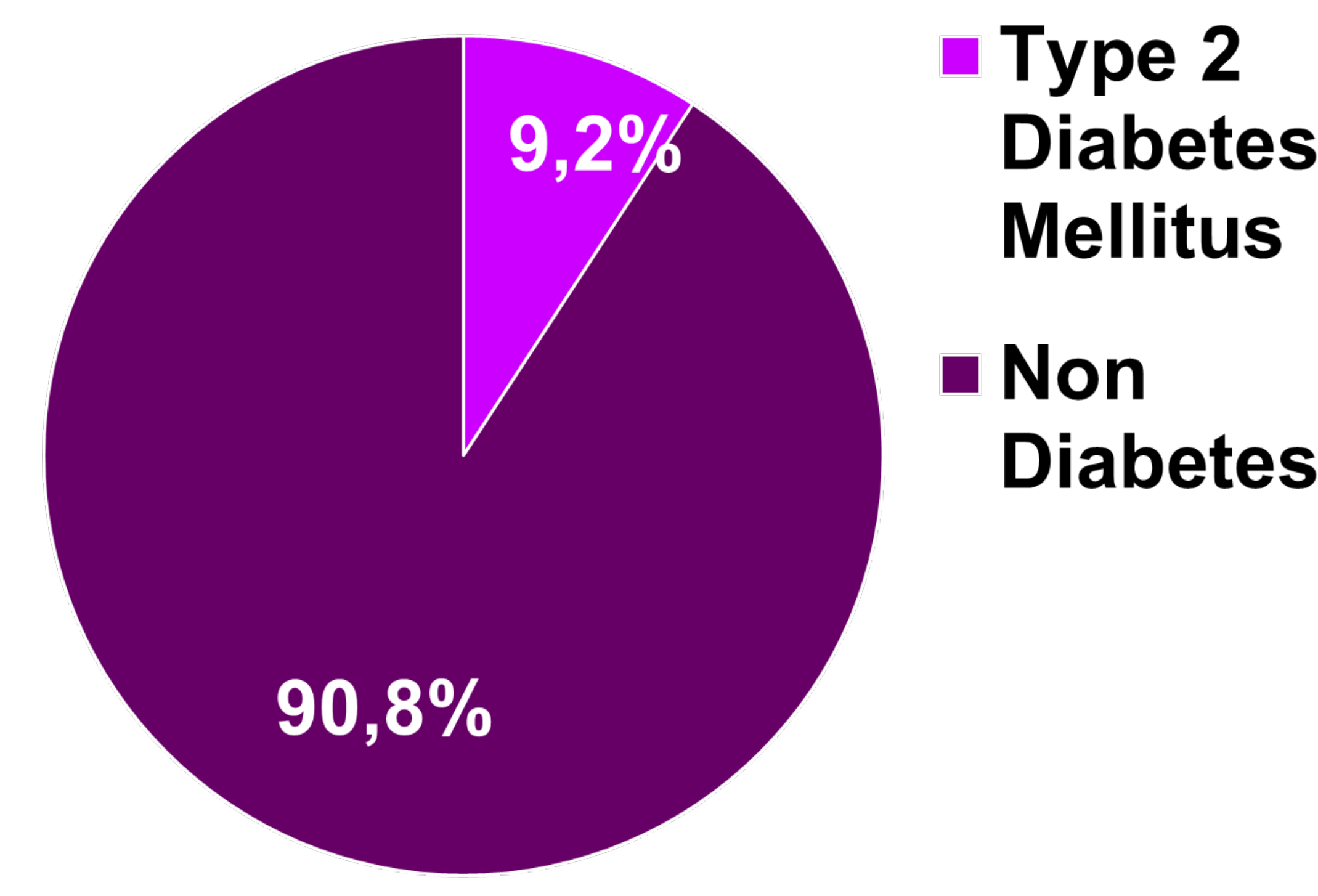

Figure 1. Prevalence of Type 2 Diabetes Mellitus in patients with Tuberculosis

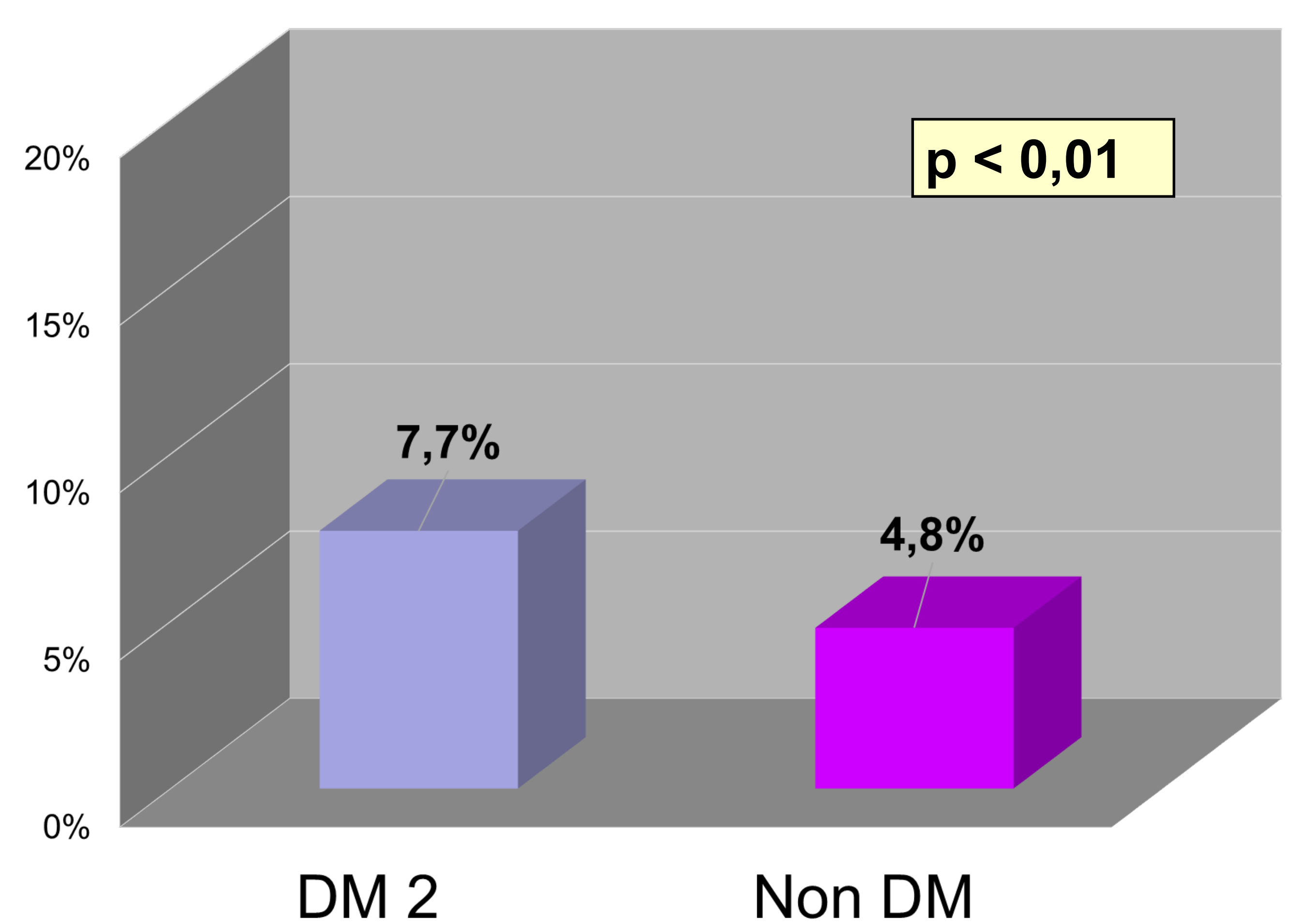

Figure 2. Mortality in patients with tuberculosis, with or whitout Type 2 Diabetes Mellitus (DM 2).

\section{Conclusions}

- In our study, TBC patients admitted to Spanish hospitals in the presence of DM2 and also those with hypoglycaemia showed an increased mortality.

- The extra-pulmonary and spread forms are also relatively frequent (>30\%) in subjects with DM2.

- Early screening of DM2 and/or treatment with tight glycemic control in individuals hospitalized for TBC might be able to decrease the mortality associated in patients with both diagnoses on admission.

- Patients with diabetes with a poor treatment adherence and a $\mathrm{HbA} 1 \mathrm{C}>6 \%$ could be a risk group for developing TBC, probably due to the long term immunosuppressive state thus generated.

- Recent studies indicate that the coexistence of DM and TB alters the distribution of the cell populations in the immune system, although large cross-sectional trials are required. 